\title{
A Short Proof that Lebesgue Outer Measure of an Interval is Its Length
}

\author{
Jitender Singh \\ Department of Mathematics, Guru Nanak Dev University Amritsar, India \\ sonumaths@gmail.com; jitender.math@gndu.ac.in
}

MSC: Primary 28A12. The Lebesgue outer measure $m^{*}(E)$ of a subset $E$ of real line is defined as $m^{*}(E):=\inf \left\{\sum_{k=1}^{\infty} \ell\left(I_{k}\right) \mid E \subseteq \cup_{k=1}^{\infty} I_{k}\right\}$, where each $I_{k}$ is an open interval and $\ell\left(I_{k}\right)$ is its length. Establishing one of the inequalities in the standard proof of the fact in the title above turns out to be tedious in [1, p. 31]. Using the connectedness of the interval shortens the proof as follows.

Proof. Given two real numbers $a$ and $b$ with $a<b$, it is enough to prove that $m^{*}([a, b])=$ $b-a$. Clearly, $m^{*}([a, b]) \leq b-a$. Now let $[a, b] \subset \cup_{k=1}^{n} I_{k}$ for some positive integer $n$, which is always possible since $[a, b]$ is compact. Without loss of generality, assume that the set $[a, b] \cap I_{k}$ is nonempty for each $k$. Observe that the set $\cup_{k=1}^{n} I_{k}$ is connected. (Otherwise, if $(P, Q)$ is its separation, then for each $k$, by connectedness of $I_{k}$, either $I_{k} \subset P$ or $I_{k} \subset Q$. Thus each of $P$ and $Q$ is equal to union of sets from the list $\left\{I_{1}, \ldots, I_{n}\right\}$. So the pair $(P \cap$ $[a, b], Q \cap[a, b])$ determines a separation of $[a, b]$, which contradicts connectedness of $[a, b]$. So $\cup_{k=1}^{n} I_{k}$ is an open interval containing $[a, b]$. Thus, $b-a \leq \ell\left(\cup_{k=1}^{n} I_{k}\right) \leq \sum_{k=1}^{n} \ell\left(I_{k}\right)$, where the last inequality holds since some intervals overlap ${ }^{\dagger}$. Hence, $b-a \leq m^{*}([a, b])$.

${ }^{\dagger}$ The inequality $\ell\left(\cup_{k=1}^{n} I_{k}\right) \leq \sum_{k=1}^{n} \ell\left(I_{k}\right)$ can be justified as follows. Given a bounded interval $I$, by definition, $\ell(I)=\sup I-\inf I$. Observe that if two distinct bounded intervals $I_{1}$ and $I_{2}$ overlap, then $\ell\left(I_{1} \cup I_{2}\right) \leq \ell\left(I_{1}\right)+\ell\left(I_{2}\right)$.

Given $n>1$ bounded open intervals $I_{1}, \ldots I_{n}$ with their union being connected implies that for each $k=1, \ldots, n-1$ we may choose $I_{k}$ after re-indexing these intervals, such that $\cup_{j=1}^{k} I_{j}$ is connected and it overlaps at least one interval among rest of the $(n-k)$ intervals, which we denote by $I_{k+1}$. So, we have $\ell\left(\cup_{k=1}^{n} I_{k}\right)=\ell\left(\cup_{k=1}^{n-1} I_{k} \cup I_{n}\right) \leq \ell\left(\cup_{k=1}^{n-1} I_{k}\right)+\ell\left(I_{n}\right) \leq$ $\ell\left(\cup_{k=1}^{n-2} I_{k}\right)+\ell\left(I_{n-1}\right)+\ell\left(I_{n}\right) \leq \ldots \leq \ell\left(I_{1}\right)+\ldots+\ell\left(I_{n}\right)$.

\section{References}

[1] H. L. Royden, P. M. Fitzpatrick, Real Analysis. Fourth ed. Pearson, Boston, 2010. 